Die Herausforderung wird sein, die guten Ergebnisse in die Praxis zu übertragen. Meines Erachtens nach wird dies nur durch eine zeitnahe Evaluierung und standardisierte Behandlung potenziell immunassoziierter Nebenwirkungen möglich sein. Voraussetzung hierfür ist eine detaillierte Aufklärung der Patienten über mögliche Symptome.

Was wird die Zukunft bringen? In einer aktuellen Arbeit wurde Pembrolizumab, ein anderer PD1-blockierender Antikörper, als Monotherapie in 2 verschiedenen Dosierungen ohne Vergleichsarm untersucht bei 495 NSCLC-Patienten, behandelt und unbehandelt [Garon EB et al. N Engl J Med. 2015; 372(21):2018-28]. Hier erwies sich eine PD-L1-Expression von > 50\% der Tumorzellen als prädiktiv. Diese Subgruppe umfasste circa $23 \%$ der Patienten. Bei innen betrug das PFS 6,1 Monate und das mediane OS wurde noch nicht erreicht. Es ist zu berücksichtigen, dass insgesamt nur $17 \%$ der Patienten in dieser Studie ein sqNSCLC hatten, was eine zu kleine Fallzahl ist, um diese Subgruppe valide auswerten zu können. Deshalb ist aktuell ein Vergleich von Pembrolizumab und Nivolumab beim sqNSCLC nicht möglich.

Die Ergebnisse weiterer, neuer Phase-IIIZweitlinienstudien beim sqNSCLC zeigten, dass die Kombination aus Ramucirumab plus Docetaxel einer Docetaxel-Monotherapie überlegen ist (Verlängerung des OS um 1,4 Monate; HR 0,86; $p=0,023$ ) [Garon EB et al. Lancet. 2014;384(9944):665-73]. Außerdem verlängerte Afatinib das Überleben im Vergleich zu Erlotinib um 5 Wochen (HR 0,81; $\mathrm{p}=0,0077$ ) [Soria et al. Lancet Oncol. 2015;16(8):897-907]. In beiden Studi- en traten bei $>50 \%$ der Patienten höhergradige Nebenwirkungen auf.

Zusammengefasst ist Nivolumab beim sqNSCLC den zugelassenen und neueren, klinisch weit entwickelten Substanzen in Bezug auf Wirksamkeit und Verträglichkeit überlegen. Deshalb ist Nivolumab aktuell bei den Patienten ohne Kontraindikationen wahrscheinlich die beste Option für die Zweitlinientherapie. Der Stellenwert von Pembrolizumab muss noch geklärt werden.

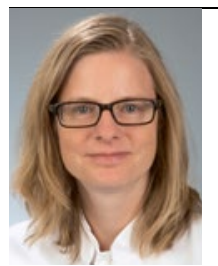

PD Dr. Dr. Sonja Loges Klinik für Onkologie, Hämatologie, KMT mit Sektion Pneumologie, Universitätsklinikum Hamburg-Eppendorf s.loges@uke.de

\section{Polymorphismen beim NSCLC bedeutsam}

\section{Bestimmte vererbte Veränderungen in Genen, die für Tumorangiogenese, Progression und Metastasierung eine Rolle spielen, könnten auch für das Therapieansprechen und die Prognose relevant sein.}

D azu gehören beispielsweise Gene für Metallomatrixproteinasen (MMP), vaskuläre endotheliale Wachstumsfaktoren (VEGF), den Fibroblasten-Wachstumsfaktorrezeptor (FGFR) und Cyclooxygenase-2 (COX-2). Polnische Onkologen untersuchten den Effekt funktioneller Polymorphismen von Genen für MMP-1, MMP-2, MMP-3, VEGF, VEGFR2, FGFR4 und COX-2 auf Gesamt-

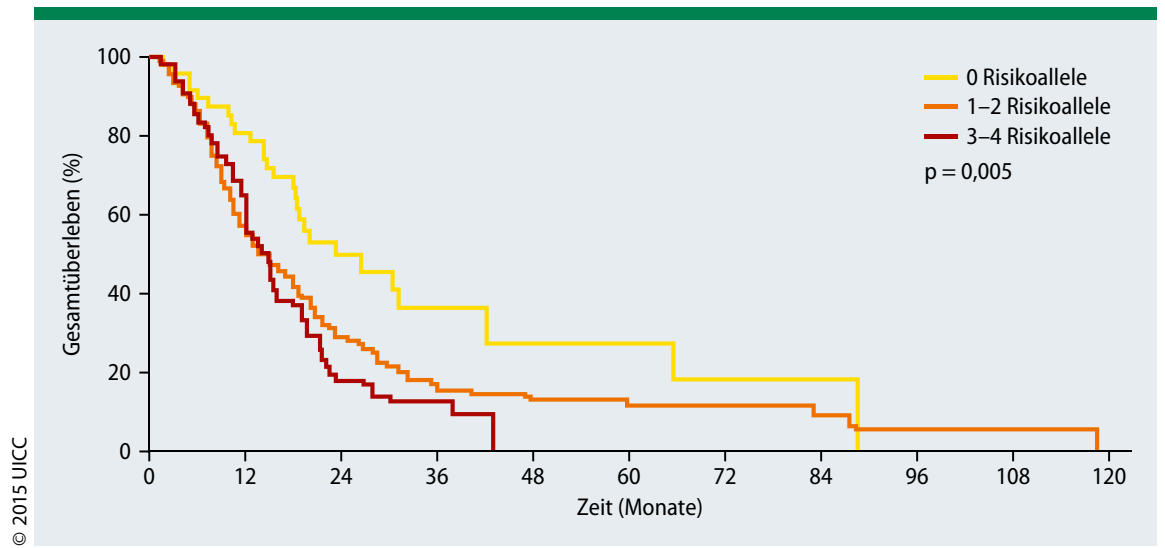

Abb. 1: Das Gesamtüberleben nimmt mit der Zunahme der Zahl von Risikoallelen ab.
(OS) und progressionsfreies Überleben (PFS) von 350 Patienten mit einem inoperablen, nichtkleinzelligen Lungenkarzinom (NSCLC). Alle Patienten unterzogen sich einer Strahlentherapie (47\% in kurativer Absicht). $67 \%$ der Patienten erhielten außerdem 1-6 Zyklen einer platinbasierten Chemotherapie. Das mediane OS lag bei 15,4 Monaten, das mediane PFS bei 10,7 Monaten. Die

mediane Beobachtungszeit betrug 33,4 Monate.

Die multivariate Analyse ergab einen deutlichen Hinweis darauf, dass die Allele VEGFR2-906C und COX-2-1195G mit einem besonders schlechten OS ( $p=0,002$ bzw. 0,015$)$ und PFS ( $p=0,009$ bzw. 0,015) assoziiert waren. Träger des Allels MMP-2-1306T wiesen ein signifikant verringertes PFS auf ( $p=0,010)$, allerdings kein reduziertes OS. Außerdem nahm das Sterbe- und Progressionsrisiko mit der Zahl ungünstiger Allele signifikant $\mathrm{zu}$ (OS: $\mathrm{p}=0,0003$; PFS: $\mathrm{p}=0,0001 ;$ Abb. 1).

Schließlich wurden außer diesen Allelen auch noch die Genotypen VEGFR2 TC/CC, COX-2 AG/GG und MMP-2 CT/ $\mathrm{TT}$ als Risikovarianten identifiziert, die unabhängig von anderen Faktoren OS und PFS ungünstig beeinflussten.

Fazit: Bestimmte Polymorphismen in den Genen für VEGFR2, COX-2 und MMP-2 könnten einzeln und in Kombination prognostisch relevante Marker beim inoperablen NSCLC sein, das mit Strahlen- oder Strahlenchemotherapie behandelt wird.

Friederike Klein

Butkiewicz D et al. The VEGFR2, COX-2 and MMP-2 polymorphisms are associated with clinical outcome of patients with inoperable nonsmall cell lung cancer. Int J Cancer. 2015. 15;137(10):2332-42. 